\title{
THE EFFECTIVENESS OF MAINTENANCE ELECTROCONVULSIVE TERAPHY
}

P. Sousa Martins ${ }^{1}$, J. Vitória-Silva1, P. Frias ${ }^{2}$, F. Caetano ${ }^{2}$, R. Valido ${ }^{3}$, S. Pimenta ${ }^{4}$, J. Mota ${ }^{1}$. ${ }^{1}$ Hospital de Magalhães Lemos- EPE, C Department, Porto, Portugal. ${ }^{2}$ Hospital de Magalhães Lemos- EPE, B Department, Porto, Portugal. ${ }^{3}$ Hospital de Magalhães Lemos- EPE, D Department, Porto, Portugal. ${ }^{4}$ Hospital de Magalhães Lemos- EPE, Intensive Intervention Service, Porto, Portugal.

\section{INTRODUCION}

The ultimate goal of maintenance electroconvulsive therapy (mECT) is remission of symptoms and prevention of relapses. This treatment option is being increasingly used in patients who completed a successful acute course of ECT . Others studies have shown a decrease in number of admission to the ward after initiation of mECT and our was objective was to reciprocate those results on our ECT unit in Porto.

- We did a retrospective mirror-image study comparing, in each patient, the number of hospital admissions and the number of days in admission before and after the initiation of $\mathrm{mECT}$.

- We collected information for demographics, diagnosis and technical data regarding ECT

- We also collected data for Fluoxetine and Olanzapine equivalents before and after treatment, to test for bias.

- We used a MECTA SpECTrum $5000 Q \circledR$ for all ECT treatments. Statistical analysis was performed under SPSS.

\section{RESULTS}

A total of 16 patients were enrolled on the study. Mean age was 53 years, $56.3 \%$ were males and the most common diagnosis was Schizophrenia. Median number of mECT treatments was 29 and median treatment duration was 23.21 months. $87.5 \%$ of patients received a bi-temporal stimulus with a median dosage of 480 millicoulombs $(\mathrm{mC})$.

\begin{tabular}{|l|c|c|c|} 
& Before mECT & After $\mathrm{mECT}$ & Wilcoxon Test \\
\hline Number of admissions & $2,0(1,5-2,5)[1,0-8,0]$ & $0,0(0,0-0,0)[0,0-4,0]$ & $\mathrm{p}=, 003$ \\
\hline Days in admission & $86,0(76,0-116,0)[32,0-206,0]$ & $14,5(0,0-55,5)[0,0-112,0]$ & $\mathrm{p}<, 001$ \\
\hline
\end{tabular}

Table 1. Before and after mECT initiation comparisons for admissions. Results presented as $\operatorname{Mdn}\left(\mathrm{P}_{25}-{ }_{75}\right)[\mathrm{Min}-\mathrm{Max}]$.

\begin{tabular}{c|c|c|c} 
& Before mECT & After mECT & Wilcoxon Test \\
\hline Fluoxetine $(n=9)$ & $36,68(28,25)$ & $49,88(27,21)$ & $\mathrm{p}=, 053$ \\
\hline Olanzapine $(\mathrm{n}=14)$ & $10,46(8,51)$ & $12,10(8,15)$ & $\mathrm{p}=, 280$
\end{tabular}

Table 2. Before and after mECT initiation comparisons for medication. Results presented as M (SD).

\section{CONCLUSION}

This study shows that mECt is a valid option for first line treatment resistant patients who have multiple admissions in the hospital. Our patients had almost no new admissions after initiation of mECT. We also had a significant cut in number of days in admission, demonstrating that these are shorter, and therefore less onerous. The biggest limitation to this study is the sample size. We hope to have a bigger database in the future, as we increase the number of patients doing $\mathrm{mECT}$ in our unit.

References:. 1. Mota, Jorge, and Nuno Rodrigues-Silva "Mirror-Image Study of Maintenance Electroconvulsive Therapy." The Joumal of ECT, vol. 32, no. 2, 2016, pp. 119-121. 2. Gupta, Susham, et al. "Efficacy of Maintenance Electroconvulsive Therapy in Recurrent Depression." The Joumal of ECT, vol. 24, no. 3, 2008, pp. 191-194. 3. National Institute for Health and Care Excellence. Guidance on the use of electroconvulsive therapy [NICE website]. 2003. Available at: 